\title{
SDN-based Handover in Future WLAN
}

\author{
Syed Mushhad M. Gilani ${ }^{1}$ * , Wenqiang Jin ${ }^{2}$, Tang Hong ${ }^{2}$, \\ Guofeng $\mathrm{Zhao}^{2}$ and Chuan $\mathrm{Xu}^{2}$ \\ ${ }^{1}$ School of Computer Science and Technology \\ ${ }^{2}$ School of Communication and Information Engineering \\ Chongqing University of Posts and Telecommunications, Chongqing, 400065, \\ China. \\ mushhad@hotmail.com*
}

\begin{abstract}
Presently, the smart devices consumers prefer IEEE 802.11-based wireless local area network (WLAN) to access the Internet due to ease of access, low cost, tremendous throughput, and provision for multimedia applications. However, traditional WLAN suffers from a critical issue regarding handover management because it has not been well-announced in IEEE 802.11 according to future Internet demands. Researchers argue that Software Defined Network (SDN) be future Internet technology revolution that brings significant impact on a wireless network. In this paper, we propose a framework within the SDN paradigm, which enables mobility management, load balancing, and position management applications on top of the centralized controller. The proposed framework implements load targeted handover (LTH) scheme for mobile stations to offers seamless handover and targeted association with underloaded APs. We evaluate LTH approach in a simulation environment then implement in real-time WLAN tested, and results demonstrate that SDN-enabled handover process leads to consistent throughput as compared to a traditional approach.
\end{abstract}

Keywords: mobility management, software defined network (SDN), WLAN, seamless handover, access point $(A P)$

\section{Introduction}

With the proliferation of smart devices, IEEE 802.11 wireless local area network (WLAN) is getting more popularity among the consumers. In the year of 2014, the number of tablet devices connection with AP has increased 1.6 fold to 74 million and 189 million laptops has accessed the Internet through WLAN [1]. In the upcoming years, 97\% smart traffic could communicate through $\mathrm{WiFi} / \mathrm{WLAN}$ devices. The rising demand for WiFi networks encourages academia and industry to develop standards for better quality of service, and enhance the mobility management features.

The existing proprietary-based WLAN architectures are manufacturer dependent that makes the innovation process reluctant and has faced several challenges to adopting future network services and applications. One of the promising challenges in existing WLAN is mobility management that relies on the access point (AP) association and handover cost. AP association complication occurs when multiple APs coverage areas are overlapped among mobile station (MS). In conventional WLAN, the AP association process typically relies on received signal strength indication (RSSI) that can lead to the load imbalanced environment [2]. Meanwhile, an MS during handover may connect with overloaded AP that can decrease the performance of associated station regarding throughput degradation, delay aspects, packet loss and in results, the connection could be lost. The another challenge of conventional IEEE 802.11WLAN-related to association schemes to considers only user selection method for connection establishment with AP 
that usually causes throughput degradation [3] and does not permit seamless mobility among adjacent APs. Although researchers have been introducing several handoff methods [4-5] for WLAN to improve the network performance. Such as selective channel scanning method [6] proposed to reduce the scanning time during new selection and prefers to connect using caching information. However, this technique can be inaccurate if an MS changes the direction in comparison to cashed events. MS position identification considers as an essential element for location-based services, and handoff decisions [7].The most appropriate method for indoor location management is WLAN localization. Researchers have introduced several positions measuring method on the basis of RSSI values of APs [8]. Network selection procedures in WLAN managed by a centralized controller to collects APs information and sends to each MS where RSSI is a key parameter for estimates the location information of end terminals [9]. Figure 1 shows, data communication, depends on the distance between MS and AP, MS near to AP in a green circle can use maximum transmission rate, and it steadily becomes slower as the MS goes farther from the associated AP [9].

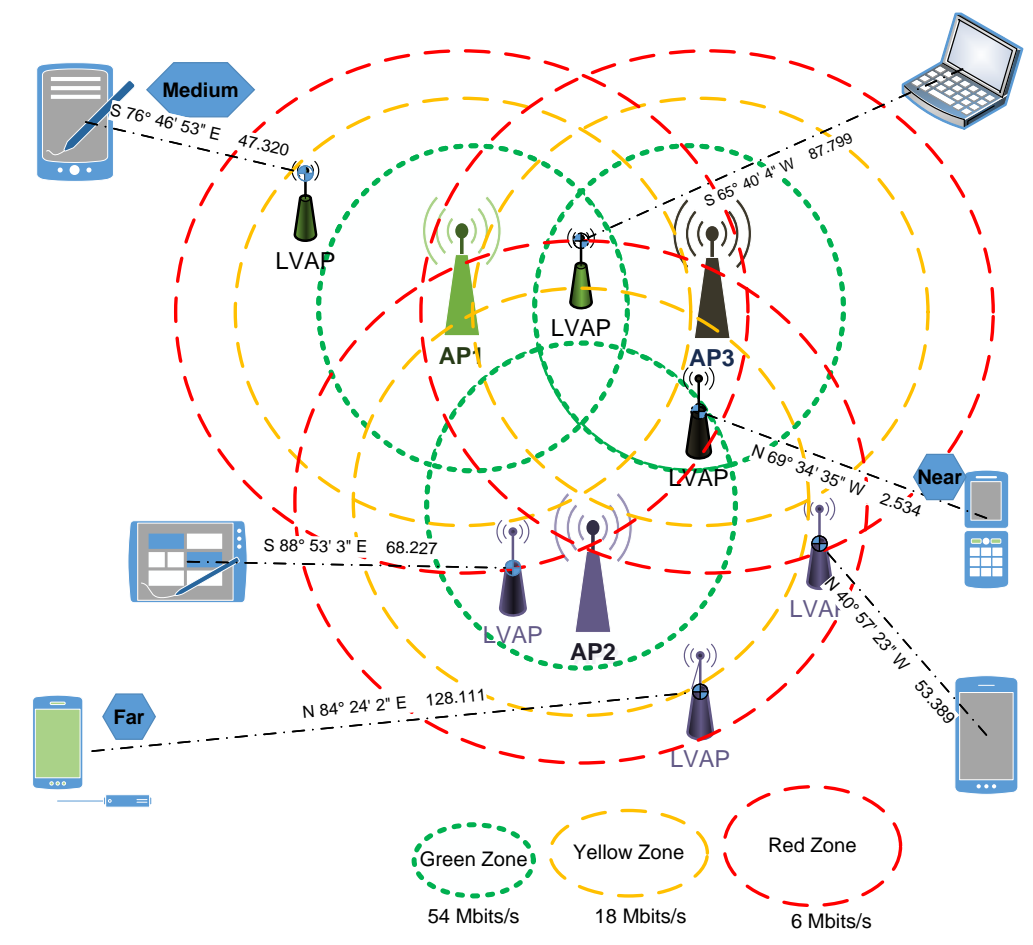

\section{Figure 1. An Example of WLAN Associated Mobile Stations with Different Bandwidth Zones According to Position}

Software Defined Network (SDN) brings innovative directions in wireless networks through the separation of control plane and data plane to provides fine-grained packet control with global access of the entire network [10]. Mobility management is a compelling aspect of a wireless network that can inherit desirable characteristics with the implication of SDN. With the advent of SDN, we proposed a framework for enterprise WLAN based on SDN paradigm to implement load targeted handover (LTH) scheme to perform handover on the basis of current traffic load with the position awareness. The proposed framework inherits the SDN data plane and control plane functionalities in which logically centralized controller monitors the access network and provides a global view of the entire network. The access network contains OpenFlow-enabled switches and APs that are based on split-MAC architecture. In proposed framework, each AP configures with a logical agent to manage local network requests, receives instructions 
from the controller, and sends local information to the controller. More specifically, the main contributions of this paper include:

- We take advantages the SDN layer-based architecture to introduce a new framework for enterprise WLAN. Northbound interface interacts with application layer which contains three major applications that are mobility management, position management, and load balancing. The SDN control layer interacts the physical layer through the southbound interface for forwarding rules and getting the updated information of wireless access network devices.

- Therefore, this paper proposes the LTH scheme in the SDN-based WLAN in order to provide seamless mobility with minimized handover delay and association with the lowest work loaded AP to maintain the constant throughput of associated MS.

- $\quad$ We target the AP association problem into following subsections.

$>\quad$ Measure the position of associated MS as illustrated in Figure 1.

$>\quad$ Calculate the traffic load (TL) of targeted APs.

$>\quad$ Association of MS with underload AP.

- We build a real-time testbed in enterprise WLAN to validate the performance of LTH scheme and make a comparison with other approaches.

The remainder of this paper is organized as follows. Section 2 provides a brief review of SDN-enabled handover in the arena of the wireless network. Section 3 gives an overview of proposed framework. The LTH scheme for an IEEE 802.11 WLAN is presented in Section 4. The evaluation of the proposed scheme is described in Section 5 by considering simulation and real-time testbed. Finally, Section 6 concludes the paper and brings some ideas for future work.

\section{Background and Related Work}

In this section, we give background and affiliated work regarding end terminal association, handover delay, and seamless handover in IEEE 802.11 based wireless network.

\subsection{Traditional Handover}

In IEEE 802.11 WLAN the initial step for handoff is scanning of new APs to establish a connection, the second process is authentication and association with target AP [11]. In general, the association procedure of IEEE 802.11 AP is a timeconsuming task due to some common parameters such as RSSI. The conventional scanning is a lengthy process that causes a delay in channel association. It could prolong the handoff process that is one reason of degradation in the quality of network services. It is essential to minimize the delay of channel scanning process to enable fast handover mechanism in WLAN. In [4] the authors proposed a scanning scheme to reduce the AP selection time. The objective was to keep track of previously scanned channels in the cache and makes the decision for next selection. However, authors do not accommodate station position information that is necessitated in case if station replaces the previous location. IEEE 802.11[12] standards have been introduced for handover management. However, those standards have not strongly achieved seamless mobility and AP association decisions. Moreover, IEEE 802.11 does not offer any system for load balancing in WLAN [13].

\subsection{SDN-based Handover}

In this subsection, we discuss the most relevant works regarding SDN in wireless networks. SDN brings modernization in the arena of a wireless network in the form of 
mobility control, load balancing, radio resources management and fine-grained packet control. Using SDN paradigm, this research work accentuates mobility services with load balancing mechanism in a wireless network. Open Networking Foundation (ONF) has been released a white paper [14] about benefits of SDN and OpenFlow. In which they consider SDN for mobility management, multi-vendor management, reliability security, intelligent traffic control and customized service platform. However, they focused only fundamental level study without in-depth reading, and practical explanation.

The first project regarding SDN in a wireless network has been presented by OpenRoads [15-16] about handover between WiFi and WiMax. They developed an OpenFlow [17] based wireless network testbed to investigate the handover cost using n-casting approach. OpenRoads project elaborates how can be consolidated OpenFlow protocol in wireless infrastructure to empower handover between different wireless networks. The concept of logical slicing [16] with virtualization of the AP becomes as virtual APs. The perception of virtual AP extended further in Odin framework [18] as a light virtual access point (LVAP). The LVAP performed with Odin agents to send information to the controller (Odin master) regarding MS association. Odin enhances the mobility features in the wireless network and offers seamless mobility from one AP to other AP. However, Odin has some limitations such as mobility scheme builds upon RSSI parameter that is not sufficient to ensure the handover accuracy for location-based services. Second, Odin does not contain parameters regarding load calculation of individual AP. In comparison, we calculate TL using the uplink and downlink traffic probability then our proposed approach can estimate the current position to calculate the current traffic load of target access points (APs).

\section{Proposed Framework Overview}

This research proposes SDN-based WLAN architecture to perform seamless handover with load balancing and position management applications which reside on the top of SDN controller as illustrated in Figure 2. The proposed WLAN framework consists of mobile stations which are accessing Internet services through OpenFlow-enabled APs under SDN controller. However, the current research effort targets the mobility management issues in enterprise WLAN. The mobile station initiates handover request which is forward to SDN controller for association decision. SDN controller analyzes the request, make a comparison and associate the MS with under-load AP in the current network environment. In this section, we describe the layers' functionality and elaborate the main components of the proposed framework.

\subsection{Application Management Layer}

This layer contains position manager, mobility manager and load balancer as applications to implement network functions through the northbound interface of SDN control layer. The position manager keeps information regarding current and previous MS positions to offer position based applications and services. Mobility Manger is a module that provides management services and abstract view of the wireless network regarding mobility control. It keeps track of station connection establishment, re-establishment, and handover decisions. The load balancer is another application that retrieves the TL of each AP and forwards to mobility manager for handover decisions. 


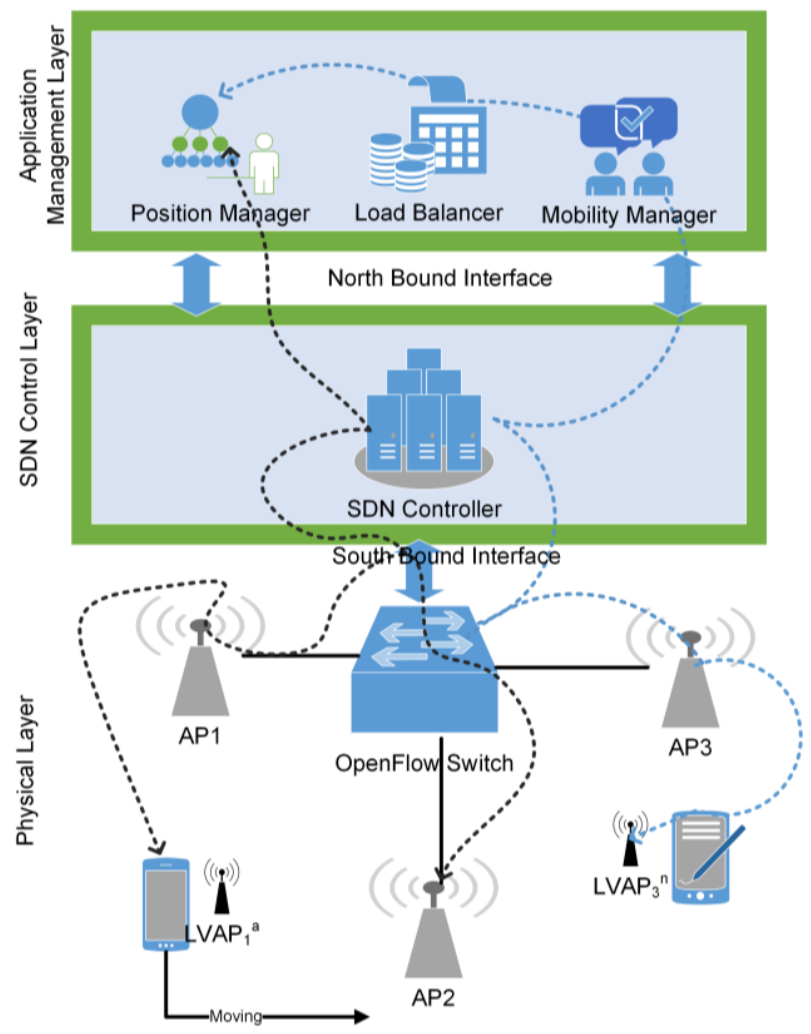

Figure 2. Proposed Framework

\subsection{SDN Control Layer}

SDN controller is an important entity of network architecture, however, if installed a single controller and in the case of a controller failure, the entire network can block, to overcome this complication, multiple replicated SDN controller approach has been adopted [19]. Furthermore, the tasks of controllers are to configure the forwarding tables dynamically for OpenFlow switch, keeps a record of each APs workloads information, collect MS position and signal quality. It also provides an interface for application management layer to controlling and viewing the entire network. In this layer, we prefer out-of-bound signaling that offers high performance with quick response especially in multimedia channels communication over WLAN. SDN controller can perform runtime traffic tagging by users location information [20].

\subsection{Physical Layer}

The core function of the physical layer is to make logical slicing of wireless network infrastructure (switch/AP) to communicate with the controller over the southbound interface. Furthermore, the physical layer is empowering the upper layers for fine-grained packet control, monitoring the flow setup, deployment of new services and functions on a physical network according to the network requirements. All APs are equipped with OpenVswitch [21] to integrate OpenFlow rules regarding traffic flow control. Besides, each AP contains mobility agent click [22] based module to receives instructions from mobility manager and collects WiFi statistics including (transmission rate, the number of LVAP, position value and TL) through TCP connection that is established between controller and AP. OpenWRT [23] is introduces reconfiguration in wireless protocols to enable programmable control plane in IEEE 802.11 AP and SDN enhance the conventional scanning process [11] in a wireless network. 


\section{Load Targeted Handover (LTH) Scheme}

In our proposed scheme centralized SDN controller collects information into two different segments as illustrated in Figure 3. The first segment is collected APs parameter which contains APs TL and a connected number of mobile stations. In an initial step, the controller accumulated APs information and announced the workload of targeted APs. The second segment is about associated MS's parameters to keep track of MS position and RSSI value. We supposed the MS position $P_{j}$ represents three position levels such as Far=1, Med=2, Near=3 as depicted in Figure 1. In the case of periodic timer expires, make a comparison with the current position, if the position is changed then perform handover optimization algorithm and choose the AP with lowest $C$ value which depends on RSSI, and TL as further detail is mentioned in section 4.3.

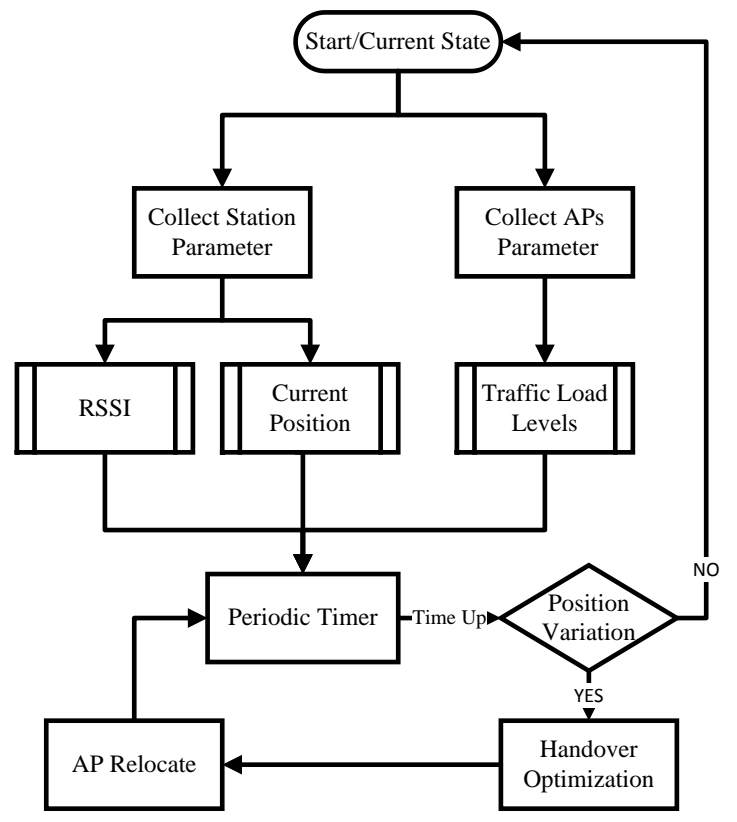

Figure 3. The Flow Diagram of LTH Scheme

\subsection{LVAP (Located Virtual Access Point)}

LVAP is a logical entity which resides in AP for each associated MS. In this research work, we extend the functionality of LVAP with addition position information parameter that could be more convenient for fast handover and also improves the AP association process to achieve load balancing in WLAN. Whenever AP receives a probe request from MS for connection establishment, forwards the request to the controller. In response to the request, the controller creates an LVAP to handle probe requests and assigned the unique BSSID. As illustrated in Figure 1, each LVAP is bind with a MS. LVAP contain parameters regarding MS information mentioned in Table 1.

Table 1. LVAP Mobile Station (MS) Tuples

\begin{tabular}{lcccc}
\hline & MAC_Address & IP Address & BSSID & Locator \\
\hline Type & String & String & String & Set \\
Size & 4 bytes & 4 bytes & 4 bytes & 4 bytes \\
\hline
\end{tabular}




\subsection{Position Determination Method}

The position determination system is based on AP coverage range and MS signal strength level. Although, several position determination schemes [24-27] has been introduced to estimate position in the wireless network. In this work to estimate the position of MS, we adopt the position estimation method that is divided into two phases [26] are described as follows.

4.2.1 Offline Training Database: In this phase, a radio finger database (RFD) for APs wireless information has been established by collecting signal values of reference access points (RAPs) on fixed positions from the perspective of different position points marked in the testbed Figure 6. Furthermore, the radio map of each RAP is calculated in assisting to build the RFD. The Radio Map of AP is shown in Figure 4(a).

Table 2. RFD Parameters

\begin{tabular}{ccc}
\hline Description & Name & Type \\
\hline Position ID & Pid & Int \\
X value & Axis x & Double \\
Y value & Axis y & Double \\
RSSI value & RSSI [ ] & Float \\
\hline
\end{tabular}

The key parameters in the RFD are mentioned in Table 2. Positions with coordinates marked in testbed $\left(x_{i}, y_{i}\right)$ are referred as $P i d_{j}$, and RSSI values received from $A P_{i}$ with different Pid $_{j}$ in the testbed are denoted as $S_{i j}$. Therefore, a map which referred as data entries in the RFD is mentioned as $\left\{A P_{i}, \operatorname{RSSI}\left[S_{i 1}, S_{i 2}, \ldots, S_{i n}\right]\right\}, i=1,2, \ldots m, j=1,2, \ldots n$ ( $m$ denoted as the number of APs, $n$ represents the number of Pid ).

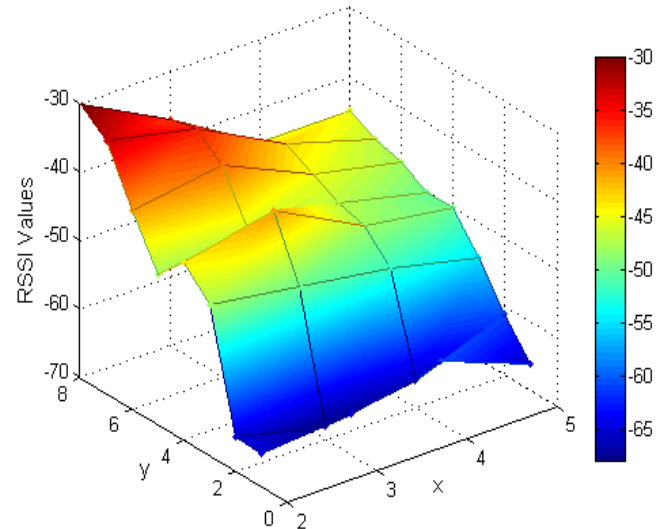

(a)

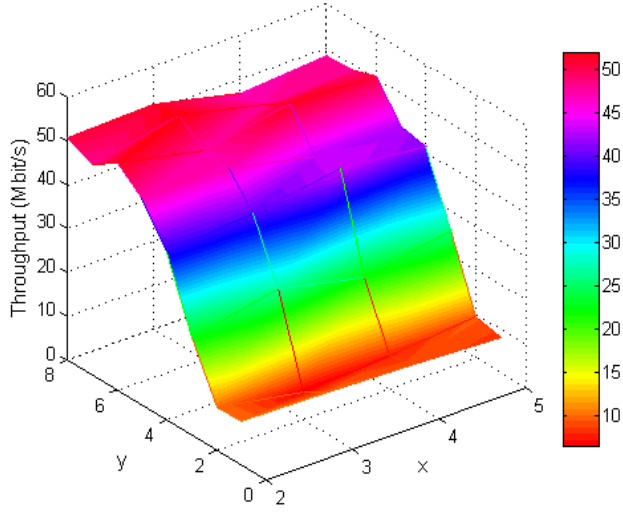

(b)

\section{Figure 4. Illustrating (a) RSSI Values and (b) Throughout, between AP and MS from Different Positions}

4.2.2 Online Position Determination: In this phase, the received wireless signal values of the terminal from various APs in its scan-able radio range are used for matching the numerical entries in RFD to determine the terminal's position as illustrated in Figure 4(b). A matching algorithm, K-Nearest Neighborhood (KNN) 
[27], is functioned to obtains the position information. Step one, the terminal which is proceeding an online position determination would acquire a signal strength value $S_{i}$ mentioned as from $A P_{i}$ by trigger active or passive scans defined in IEEE

802.11 wireless protocols. The distance between $S_{i}$ and the samples $S_{i j}$ in RFD are calculated by Equation 1 .

$$
d_{j}=\left(\sum_{i=1}^{n}\left|S_{i j}-S_{i}\right|^{2}\right)^{1 / 2} j=1,2, \ldots, n
$$

Step two, $K$ samples with relatively small values of $d_{j}$ are selected for further procedures and $\left(x_{i \mathrm{k}}, y_{i k}\right)$ is interpreted as the coordinate of $K_{t h}$ selected position samples to form the perspective $A P_{i}$. Then, by repeating the last two steps, the position of the terminal is determined by Equation 2 .

$$
(\hat{x}, \hat{y})=\left(\frac{1}{i K} \sum_{i=1}^{m} \sum_{k=1}^{K} x_{i k}, \frac{1}{i K} \sum_{i=1}^{m} \sum_{k=1}^{K} y_{i k}\right)
$$

Table 3. Notations

\begin{tabular}{cl}
\hline Symbol & \multicolumn{1}{c}{ Description } \\
\hline$A$ & Set of APs \\
$M$ & Set of all mobile stations \\
$L$ & Set of located virtual APs (LVAP) \\
$\boldsymbol{w}(\boldsymbol{l}, s)$ & Link between station s and LVAP 1 \\
$\boldsymbol{d}_{(l, s)}$ & Data rate between station $s$ and LVAP 1 \\
$P_{s}^{i}$ & Probability between station s and AP i \\
$a_{s}$ & Station $s$ associate with AP $a$ \\
$M_{a}$ & Set of mobile stations associated with AP $a$ \\
$\boldsymbol{T P}_{\boldsymbol{d}}$ & Downlink traffic probability \\
$\boldsymbol{T P}_{u}$ & Uplink traffic probability \\
\hline
\end{tabular}

\subsection{Handover Optimization Model}

One way is to promote the handover performance in dense WLAN to calculate the TL of each AP before MS association. Let set A denote the set of OpenFlowenabled APs and set of mobile stations M. Each AP $a \in A$ have installed SDN agent to creates LVAPs on association request generates by either client or SDN controller to gives the illusion of own AP for each associated client. The set of LVAPs $L$ is the subset of AP $a$ represent as $L \subset a$.A wireless link between LAP $l \in L$ and station $s \in M$ is denoted as $w(l, s)$.

$$
w(l, s)= \begin{cases}1 & \text { if } \text { station associate } \\ 0 & \text { otherwise }\end{cases}
$$

We prefer to adopt rate adaptation scheme which deliberately chooses the best modulation and coding scheme (MCS). Each link PHY rate computed by MCS that is denoted as $d_{(l, s)}$. We supposed variable $T P_{d}$ and $T P_{u}$ that represents downlink traffic probability and uplink traffic probability between client $s$, and LVAP $1 T L$ can be determined using uplink and downlink traffic.

Input for station $s$ : Set of probable AP

$$
\left\{P_{s}^{i}, P_{s}^{j}, P_{s}^{x}\right\} \quad i, j, x \in A \quad s \in M
$$

Where $P_{s}^{j}$ is RSSI probability between station $s \in M$ and AP $i \in A$ 
Here, we discuss the set of constraints. For $a$ client $s$ that associate with an AP $a$ denotes as $a_{s} \in \mathrm{A}$.

$$
\begin{gathered}
M_{a}=\left\{s \mid s \in M, a_{s}=a\right\} \quad s \in M \quad a \in A \\
\sum_{l \in L} w(l, s) \leq 1 \quad \forall s \in M \\
\sum_{s \in M} A(a, s)=\sum_{s \in M} \sum_{L \in a} L(l, s) \quad \forall a \in A \quad \forall l \in L \\
d_{(a, s)} \geq 0 \quad a \in A \quad s \in M \\
d_{(l, s)} \geq 0 \quad l \in L \quad s \in M \\
d_{(l, s)}=W_{(l, s)} T L_{(l, s)}
\end{gathered}
$$

Equation 3 represents the set $M_{a}$ where mobile stations $M$ are associated with AP $a$. Whether the user successfully associates with current AP, then Equation 4 ensures that maximum one LVAP assigned to one station. Equation 5 confirms the number of LVAP is equal to some stations connected to that AP. Equation 6 represents data rate between the station $s$ and AP $a$ or LVAP either could be equal to zero in the case of idle station or according to the current transmission rate of the station.

$$
\begin{aligned}
& T P_{u} \geq \sum_{s \in M} w(s, j) T L(s, j) \quad s \in M \quad j \in A \\
& T P_{d} \geq \sum_{s \in M} w(j, s) T L(j, s) \quad j \in A \quad s \in M
\end{aligned}
$$

Total $_{\mathrm{j}}=T P_{\mathrm{dj}}$ and $T P_{\mathrm{uj}}$

Equation 7 and 8 elaborate the estimated uplink and downlink traffic probability between station $s$ and AP $j$. However, 802.11 WLAN channel attenuation of uplink and downlink transmission between AP and client are almost same [3].

$$
\begin{aligned}
& B_{j}=\text { Total }_{j}-\text { RSSI }_{j} \quad j \in A \\
& C_{i}=\left\{B_{i}-B_{j} \mid \quad i, j \in A \quad i \neq j\right\}
\end{aligned}
$$

The objective is to find the maximize $c_{i}$ where $i$ is the best AP. Load balancer application running on top of SDN controller can announce the current TL of targeted AP before new association. On the basis of load comparison of targeted APs, the controller can take a decision about MS association. This approach enables load balancing environment between APs.

\section{Experimental Results and Discussions}

In order to demonstrate the competence of the proposed approach, numerous measures have been performed. First, we performed proposed approach in a simulation environment to exhibits by obtaining the position of MS and $C_{i}$ value of APs using Equation 10. Second, we developed a real-time WLAN testbed to evaluate the proposed approach and make a comparison with traditional and OpenFlow-based handover scheme.

\subsection{Simulation Experimental Results}

The proposed scheme has been assessed using simulation environment for testing the LTH significance. In the simulation, APs programmed to forward different background traffic. Furthermore, a terminal is selected to move through the entire simulation blueprint. Moreover, we assume that AP1 is low workload, AP2 is high workload, and AP3 is transferring moderate amounts of data. The detail simulation settings are given in Table 4. 
Table 4. Simulation Parameter Settings

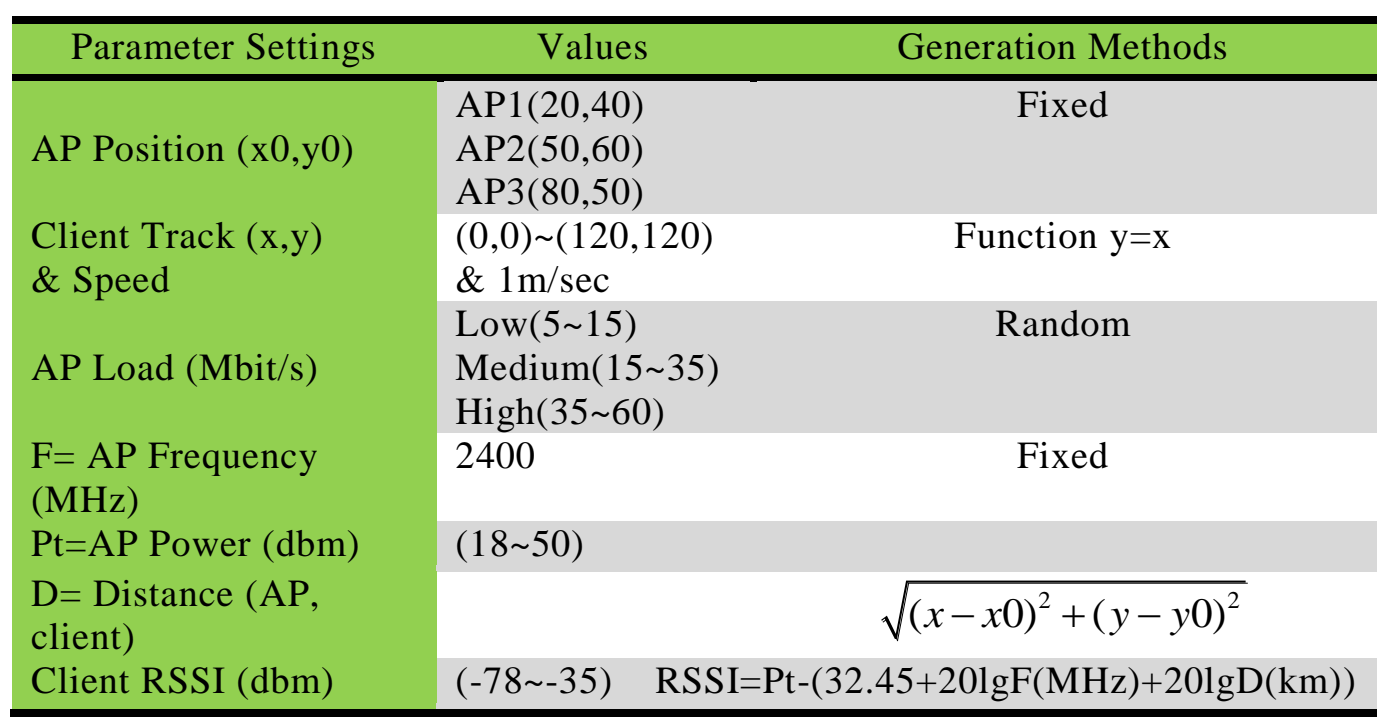

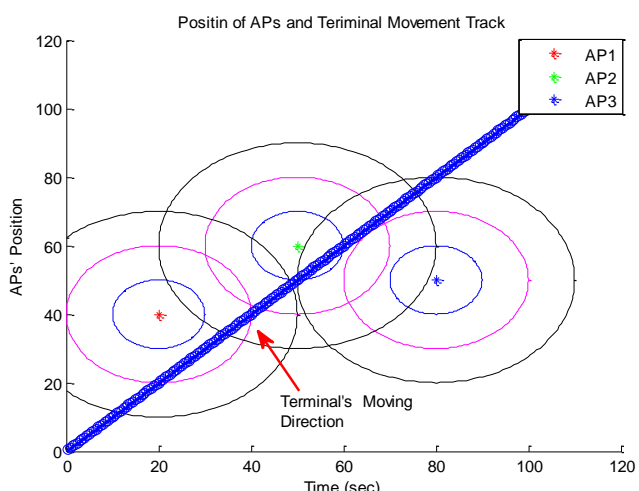

(a)

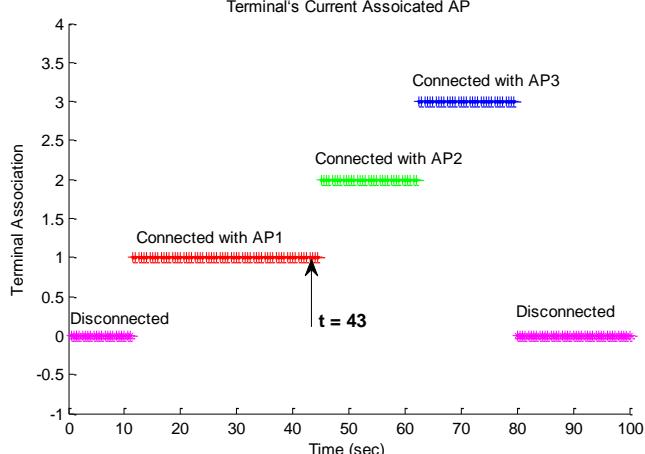

(c)

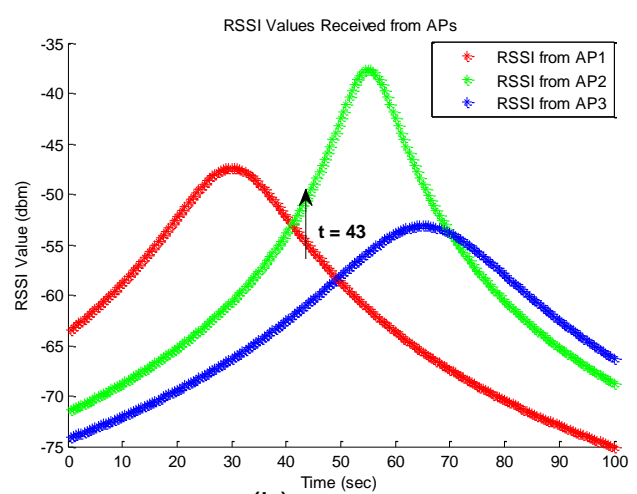

(b)

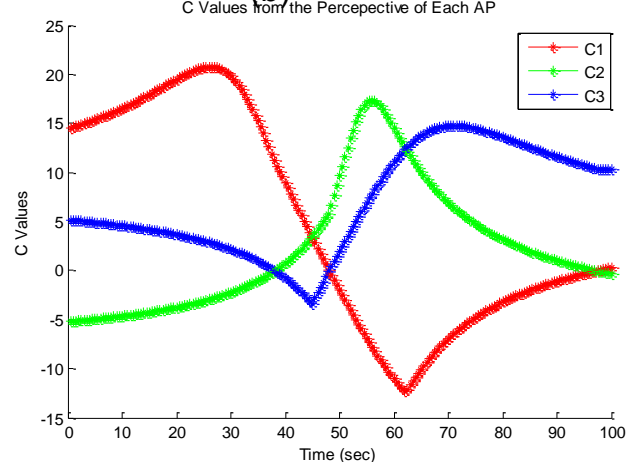

(d)

Figure 5. Simulation Results

Figure 5(a) denotes the position of three APs showing its' signal covering ranges with different qualities of wireless transmission conditions. Specifically, the blue circles cover the radio area with a prospectively good performance in wireless transmission. The area scattered between the range of blue circles, and pink circles refer a medium radio condition for terminals accessing and packets forwarding and the black circles combined with pink circles forming an area denoting relatively poor qualities in wireless transmission. During the simulation period, the terminal is 
traveling through the different radio covering ranges of three APs with a constant speed rate. Moreover, the moving track is emphasized by using a bold line.

Figure 5(b) reports the signal strengths the terminal received from each AP while it is moving across the APs radio covering areas. The RSSI lines show a consistently predictable way. When the terminal is moving toward the AP, it would receive a strong RSSI value from that AP and while it is apart from the AP, the obtained RSSI value would decrease.

Figure 5(c) reports the association condition of the moving terminal with respect to RSSI. We observed the terminal is allocated to AP1 at $t=43 \mathrm{~s}$. However, the RSSI value receiving from AP2 is obviously larger than AP1. The reason lies to that we calculated the load of each AP, the terminal's current position as well as the radio signal strength by using our proposed approach. Moreover, the obtained $C$ values according to APs are shown in Figure 5(d).

\subsection{Testbed Experimental Results}

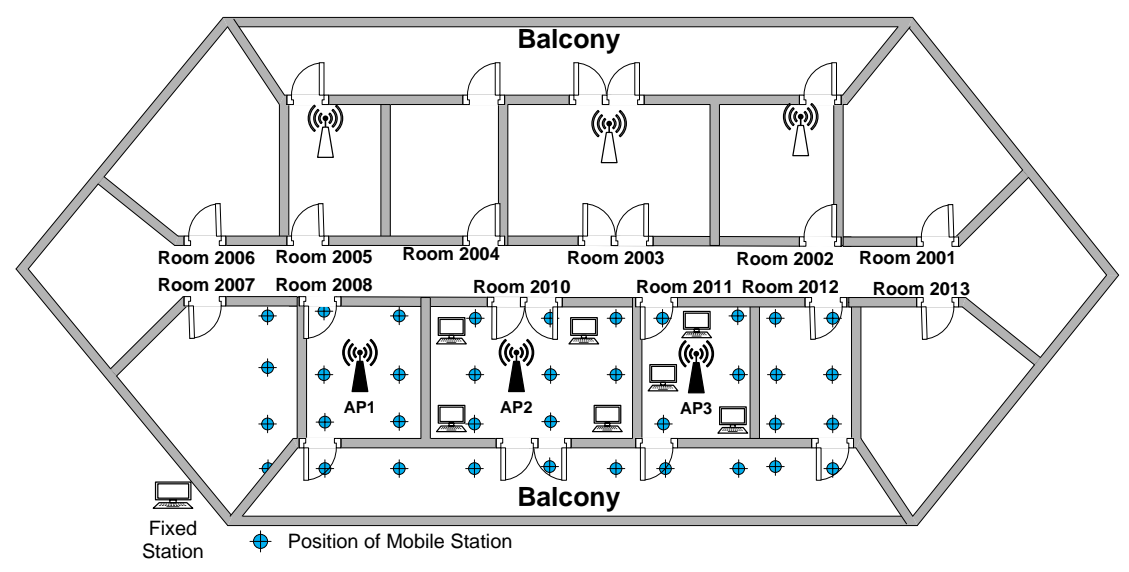

Figure 6. Testbed Setup for Handover Experiments

We have been evaluated the performance of the SDN-based handover in the real network at the $20^{\text {th }}$ floor of Xinke building in CQUPT campus. As illustrated in Figure 6, AP1, AP2, and AP3 have deployed at different positions in a closed region. The location symbol represented those points from where we collected transmission rate and RSSI readings. We have conducted two experiments. The first experiment was performed on three APs. i.e., there were no stations connected before the start of the experiment, so no background traffic was available. The objective of this experiment was to investigate the throughput during handover. The second experiment (following the first experiment setup) was conducted during peak traffic hours (when APs were loaded) to study the effect of LTH scheme. Moreover, the LTH approach is compared with conventional RSSI based algorithm and OpenFlow- enabled schemes such as Odin [18].

5.2.1 First Experiment: The goal of this experiment is to demonstrate that how LTH scheme can enhance the network performance regarding UDP and TCP throughput during handover. We performed this experiment during late night hours when there were no other users connected and make a comparison between traditional approach, Odin approach, and proposed approach. This experiment consisted of 3 APs and 3 MS's that first executed for UDP-based applications. We selected MS1 that was associated with AP1 using traditional RSSI based scheme at $15 \mathrm{Mbits} / \mathrm{s}$ throughput level. Then we started a random walk between AP1, AP2, and AP3 as illustrated in Figure 6 . At time $t=5 \mathrm{sec}$ first 
handover initiate and in results throughput decreased and touched the zero throughput level. At $\mathrm{t}=10 \mathrm{sec}$ throughput recovered and raised up to $15 \mathrm{Mbits} / \mathrm{s}$ as depicted in Figure $7(\mathrm{a})$.

The same experiment we performed using Odin based approach that reveals there was no throughput degradation during handover, and maximum UDP throughput was up to 15 Mbit/s and lower throughput was $13 \mathrm{Mbits} / \mathrm{s}$. Then we selected our proposed approach and performed this experiment in the same environment, and results are shown in Figure 7(a) that there was no throughput degradation during handover, and average UDP throughput was $15 \mathrm{Mbits} / \mathrm{s}$ that was more stable as compared with Odin.

To further understand the advantage of proposed approach we selected TCP-based application and performed the same experiment. Figure 7(b) presents, the proposed approach significantly reduced the handover delay and minimized the packet loss during handover and achieved the constant throughput level. We further evaluated the data transmission delay as illustrated in Figure 8(a). In our proposed scheme the average latency rate is $20 \mathrm{~ms}$, and Odin based approach confronted the high variations between $30 \mathrm{~ms}$ and $10 \mathrm{~ms}$ latency. The results showed that the proposed approach minimized the latency fluctuations as a comparison of Odin based approach.

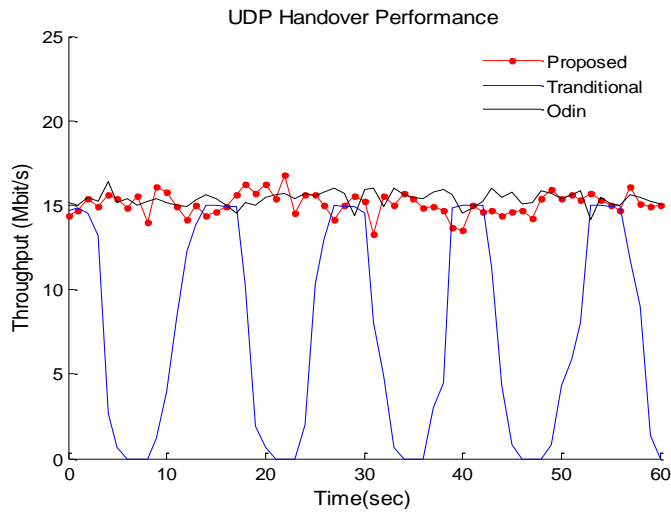

(a)

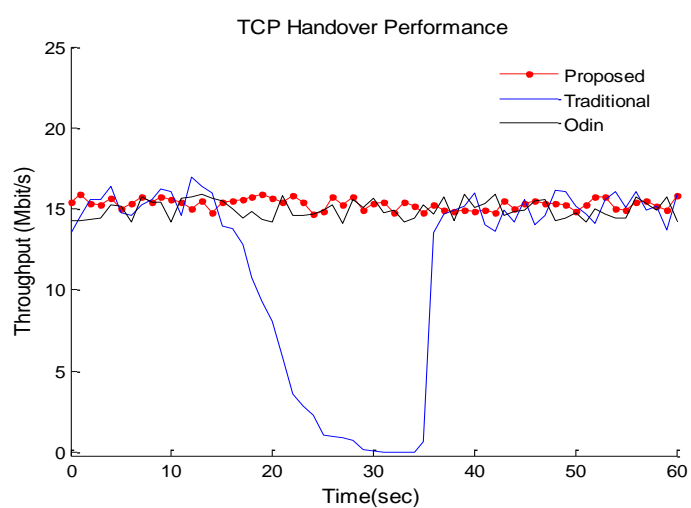

(b)

Figure 7. Throughput Performance of MS during Handover

Table 5. Impact of Background Traffic on LTH Scheme

\begin{tabular}{|l|c|c|c|}
\hline Background Traffic & $20 \mathrm{Mbit} / \mathrm{s}$ & $30 \mathrm{Mbit} / \mathrm{s}$ & $40 \mathrm{Mbit} / \mathrm{s}$ \\
\hline Uplink Data Rate (Avg.) & $40.1 \mathrm{Mbit} / \mathrm{s}$ & $32.5 \mathrm{Mbit} / \mathrm{s}$ & $26.3 \mathrm{Mbit} / \mathrm{s}$ \\
\hline Downlink Data Rate (Avg.) & $26.9 \mathrm{Mbit} / \mathrm{s}$ & $23.7 \mathrm{Mbit} / \mathrm{s}$ & $18.4 \mathrm{Mbit} / \mathrm{s}$ \\
\hline Packet Loss & $0.2 \%$ & $0.4 \%$ & $0.5 \%$ \\
\hline Round-Trip Delay (Avg.) & $6.753 \mathrm{~ms}$ & $11.185 \mathrm{~ms}$ & $25.680 \mathrm{~ms}$ \\
\hline UDP Jitter (Avg.) & $1.023 \mathrm{~ms}$ & $1.468 \mathrm{~ms}$ & $2.0367 \mathrm{~ms}$ \\
\hline
\end{tabular}

5.2.2 Second Experiment: This experimental setup was composed of three OpenFlowenabled APs with sharing signal interference area as illustrated in Figure 1. We accommodate a large number of mobile stations to analyze the load balancing between APs. We examine the performance of proposed approach with background traffic that is divided into three intensities. Each has a different impact on network performance as mentioned in Table 5. The network throughput frequency depends on current TL and background traffic. Background traffic supports to distinguish the effect of throughput degradation in this experiment. The increase in background traffic results decreases the uplink and downlink data rate while it has little effect on packet loss ratio. 
In this experiment, we have implemented LTH scheme that includes current TL, the number of associated stations and current position for client association. Whenever new association request receives, it forwards to the SDN controller for decision either association granted with targeted AP or redirect to other AP. The current position of MS information supports controller to find surrounding APs for the association. Before association, the controller calculates the throughput of MSs from different positions. We compared the proposed approach with Odin [18] that did not calculate TL before association that could be associate MS with overloaded AP, and in results, data transmission rate is reduced as mentioned in Figure 8(b).

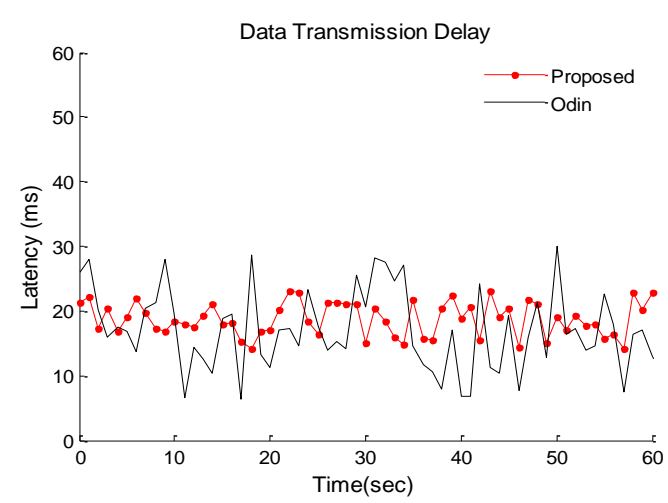

(a)

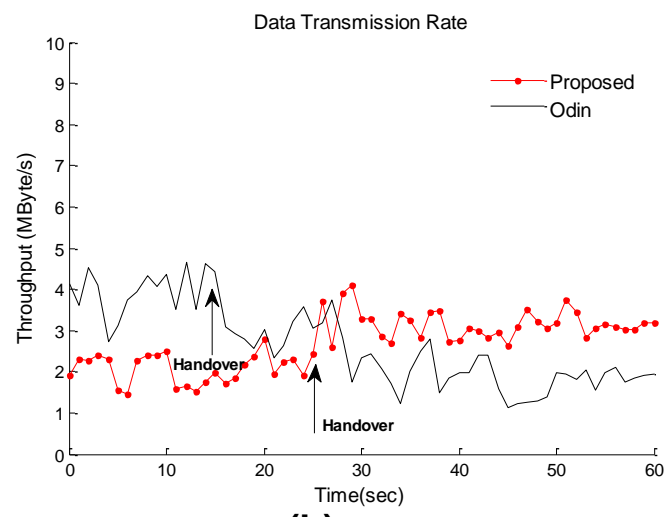

(b)

Figure 8. Data Transmission Delay and Data Rate Comparison between proposed Approach and Odin

\section{Conclusions and Future Work}

SDN introduces as future technology paradigm to enhance programmability, flexibility, scalability, interoperability and centralized control within the network. In this paper, we have examined the implications of SDN to enhance the mobility management in enterprise WLAN. We have proposed an efficient approach to incorporate the AP load with position awareness into handover procedure. We build a simulation environment to analyze the performance of handover optimization model. Afterward, the proposed framework is evaluated in the real-time network testbed, which provides assurance to achieve the configuration and controlling of OpenFlow-enabled devices (AP/Switch/Controller) in an enterprise WLAN. The experiment results shown that the proposed scheme significantly reduced the handover delay without packet drop as compared with 802.11 traditional handover scheme and also minimized the latency rate in comparison to Odin approach. The practical implementation of the proposed scheme is feasible as no modification requires to existing WLAN clients. In the future, we plan to implement proposed scheme on the larger wireless network with future WLAN applications such as location based services and traffic offloading.

\section{Acknowledgments}

This work was supported by Prospective Research Project on National Nature Science Foundation (61402065, 61501075), Future Networks (BY2013095-2-03) fund by Jiangsu Future Networks Innovation Institute and Project Foundation of Chongqing Municipal Education Committee (No. KJ1500429). 


\section{References}

[1] Global Mobile Data Traffic Forecast Update 2014-2019, White Paper CISCO, (2011).

[2] D. Gong and Y. Yang, "On-Line AP Association Algorithms for 802.11n WLANs with Heterogeneous Clients," IEEE Trans. Comput., vol. 63, no. 11, (2014), pp. 2772-2786.

[3] T. Hu, K. Xue, W. Wei and W. Jiang, "LENV: A new light-weighted edge network virtualization framework in software-defined wireless networks," in Wireless Communications \& Signal Processing (WCSP), 2015 International Conference on, (2015), pp. 1-6.

[4] S. Shin, A. G. Forte, A. S. Rawat and H. Schulzrinne, "Reducing MAC layer handoff latency in IEEE 802.11 wireless LANs," in Proceedings of the second international workshop on Mobility management \& wireless access protocols, (2004), pp. 19-26.

[5] S. H. Park, H. S. Kim, C. S. Park, J. W. Kim and S. J. Ko, "Selective channel scanning for fast handoff in wireless LAN using neighbor graph," in Personal Wireless Communications, (2004), pp. 194-203.

[6] H. Wu, K. Tan, Y. Zhang and Q. Zhang, "Proactive scan: Fast handoff with smart triggers for 802.11 wireless LAN," in INFOCOM 2007. 26th IEEE International Conference on Computer Communications. IEEE, (2007), pp. 749-757.

[7] H. Moura, G. V. Bessa, M. A. Vieira and D. F. Macedo, "Ethanol: Software Defined Networking for 802.11 Wireless Networks," in Integrated Network Management (IM), 2015 IFIP/IEEE International Symposium on, (2015), pp. 388-396.

[8] U. Grossmann, S. Gansemer and O. Suttorp, "RSSI-based WLAN indoor positioning used within a digital museum guide," Int. Journal Comput., vol. 7, no. 2, (2014), pp. 66-72.

[9] H. J. Shin, M. Jeon and C. H. On, "Network Selection Algorithm at the Starting Point of Mobile Device in Indoor Wi-Fi Environment," Int. Journal Control Autom., vol. 7, no. 9, (2014), pp. 97-108.

[10] N. A. Jagadeesan and B. Krishnamachari, "Software-Defined Networking Paradigms in Wireless Networks: A Survey," ACM Comput. Surv., vol. 47, no. 2, (2014), pp. 1-11, Nov.

[11] A. Mishra, M. Shin and W. Arbaugh, "An empirical analysis of the IEEE 802.11 MAC layer handoff process," ACM SIGCOMM Comput. Commun. Rev., vol. 33, no. 2, (2003), pp. 93-102.

[12] E. Piri and K. Pentikousis, "IEEE 802.21: media independent handover services," Internet Protoc. J., vol. 12, no. 2, (2009), pp. 7-27.

[13] M. Collotta, G. Pau, V. M. Salerno and G. Scata, "A distributed load balancing approach for industrial IEEE 802.11 wireless networks," in Emerging Technologies \& Factory Automation (ETFA), 2012 IEEE 17th Conference on, (2012), pp. 1-7.

[14] ONF White Paper, Software-defined networking: The new norm for networks, (2012).

[15] K. K. Yap, "Lossless Handover with n-casting between WiFi-WiMAX on OpenRoads," ACM Mobicom Demo, vol. 12, no. 3, (2009), pp. 40-52.

[16] K. K. Yap, "OpenRoads: Empowering research in mobile networks," ACM SIGCOMM Comput. Commun. Rev., vol. 40, no. 1, (2010) pp. 125-126.

[17] ONF http://www.opennetworking.org/.

[18] L. Suresh, J. Schulz-Zander, R. Merz, A. Feldmann and T. Vazao, "Towards programmable enterprise wlans with odin", in HotSDN'12 Helsinki, Finland, Copyright ACM, (2012), pp. 115-120.

[19] R. Ahmed and R. Boutaba, "Design considerations for managing wide area software defined networks," Commun. Mag. IEEE, vol. 52, no. 7, (2014), pp. 116-123.

[20] N. McKeown et al., "OpenFlow: enabling innovation in campus networks," ACM SIGCOMM Comput. Commun. Rev., vol. 38, no. 2, (2008), pp. 69-74.

[21] Open vSwitch http://openvswitch.org/.

[22] Click modular router project. http://www.read.cs.ucla.edu/click/click.

[23] OpenWrt http://wiki.openwrt.org/doc/start, (2004).

[24] F. Shang, W. Su, Q. Wang, H. Gao and Q. Fu, "A Location Estimation Algorithm Based on RSSI Vector Similarity Degree,” Int. Journal Distrib. Sens. Netw., vol. (2014), pp. 1-22.

[25] X. Zhu and Y. Feng, "RSSI-based Algorithm for Indoor Localization," Commun. Netw., vol. 5, no. 2, (2013), pp. 37-42,.

[26] D. Sánchez-Rodríguez, P. Hernández-Morera, J. Quinteiro and I. Alonso-González, “A Low Complexity System Based on Multiple Weighted Decision Trees for Indoor Localization," Sensors, vol. 15, no. 6, (2015), pp. 14809-14829.

[27] Y. Chen, Z. Liu, Y. Li and Y. Wang, "Indoor Localization Algorithm for Wireless Sensor Network Based on Range-Free," in Advances in Wireless Sensor Networks, Springer, (2014), pp. 221-230. 


\section{Authors}
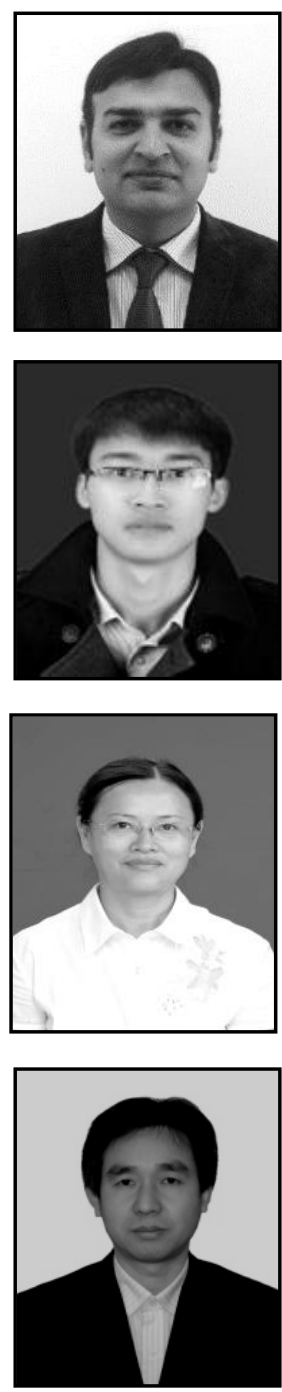

Guofeng Zhao, he received his Ph.D. degree from the Univ. of Chongqing in 2003, China. Now he serves as a professor in the Institute of Information and Network Engineering, and the director of the Research Center of Future Internet (RFI) at CQUPT. His research interests are in Future Internet, Mobile Internet, Network Management, Network Security. 
International Journal of Future Generation Communication and Networking Vol. 9, No. 12 (2016) 